\title{
Dynamics in Nonlinear Schrödinger Equation with dc bias: From Subdiffusion to Painlevé Transcendent
}

\author{
A. Iomin * \\ Department of Physics and Solid State Institute, \\ Technion - Israel Institute of Technology, Haifa, 32000, Israel
}

\begin{abstract}
Dynamics of the nonlinear Schrödinger equation in the presence of a constant electric field is studied. Both discrete and continuous limits of the model are considered. For the discrete limit, a probabilistic description of subdiffusion is suggested and a subdiffusive spreading of a wave packet is explained in the framework of a continuous time random walk. In the continuous limit, the biased nonlinear Schrödinger equation is shown to be integrable, and solutions in the form of the Painlevé transcendents are obtained.
\end{abstract}

Keywords and phrases: continuous time random walk, subdiffusion, fractional FokkerPlanck equation, Stark ladder, self-accelerating solution

Mathematics Subject Classification: 60J25, 60H30, 60J60

\section{Introduction}

It is well known that wave propagation in random media can be described in the framework of the FokkerPlanck equation, under certain conditions [37]. In modern optical experiments with nonlinear media a more suitable description is developed in the framework of the fractional kinetics based on fractional integro-differentiation. This concept of differentiation of non-integer orders arises from works of Leibniz, Liouville, Riemann, Grunwald, and Letnikov, see e.g., [34,38]. Its application is related to random processes with power law distributions. This corresponds to the absence of characteristic average values for processes exhibiting many scales $[27,31]$.

A typical example of fractional dynamics in optics is realized in a competition between localization and nonlinearity that leads to anomalous transport $[11,15,30,36,40]$. This dynamics is described in the framework of the nonlinear Schrödinger equation (NLSE) in the presence of an external field $V=$ $V(x), x \in(-\infty,+\infty)$. The wave spreading, described by the wave function, is governed by

$$
i \partial_{t} \psi=-\partial_{x}^{2} \psi+\beta|\psi|^{2} \psi+V \psi,
$$

where $\beta$ is a nonlinearity parameter. The variables are chosen in dimensionless units and the Planck constant is $\hbar=1$. In the case of a random potential, $V(x)$ is such that, in the linear case $(\beta=0)$, the Anderson localization takes place $[1,23]$, and the system is described by the exponentially localized

*Corresponding author. E-mail: iomin@physics.technion.ac.il 
Anderson modes (AM)s $\Psi_{\omega_{k}} \equiv \Psi_{k}(x)$, where $\Psi_{\omega_{k}}(x)$ are real functions and the eigenspectrum $\omega_{k}$ is discrete and dense [23]. This problem is relevant to experiments in nonlinear optics, for example, disordered photonic lattices [22,39], where Anderson localization was found in the presence of nonlinear effects, as well as to experiments on Bose-Einstein condensate released into a one-dimensional waveguide in the presence of a controlled disorder [6]. The nature of this anomalous transport is chaotic motion inside the multidimensional separatrix network $[7,29,33]$.

Another illuminating example of subdiffusion, described by the NLSE in an optical lattice, corresponds to a discrete nonlinear Stark ladder [20,21], which is characterized by nonlinearity $\beta$ and the dc electric field $E$. In dimensionless variables, it reads $[20,21]$

$$
i \dot{\psi}_{n}=-\left(\psi_{n+1}+\psi_{n-1}\right)+n a E \psi_{n}+\beta\left|\psi_{n}\right|^{2} \psi_{n},
$$

where $a$ is a period of the lattice. Contrary to a random potential, the discreteness here is crucial for the realization of localization, which is obtained in the linear part of the equation and, consequently, the discreteness is important for chaotic dynamics and subdiffusion $[20,21]$. When $\beta=0$, the eigenvalues correspond to the Stark ladder with the spectrum $\omega_{m}=m E$, where $m \in \mathbb{Z}$ are integers. The eigenfunctions, or normal modes, obey the functional equation for the Bessel functions of the first kind $[9,12]$ $J_{n+1}+J_{n-1}=\frac{2 n}{z} J_{n}(z)$, where $z=2 / E$.

In this paper, we study both the continuous and discrete limits of the NLSE (1.2), where the dynamics between the limits changes drastically. The analysis of subdiffusion coincides exactly with that of Eq. (1.1), since the linear parts of both equations yield Anderson localization, and we follow the analysis of Refs. $[15,16]$ developed for the NLSE in random potential.

The situation with the continuous limit of Eq. (1.2) differs radically. First of all, the linear part is described by the Airy function, and localization is absent. The continuous NLSE is integrable and corresponds to the second Painlevé transcendent, expressed by expansion $\sum_{i=1}^{\infty} a_{i}\left(x-x_{0}\right)^{i}$ in the vicinity of a regular point $x_{0}[8]$. We also note that this limit corresponds to experimental realizations of selfaccelerating optical beams in highly nonlocal and nonlinear media [3]. This finding also corresponds to a special solution of the Schrödinger equation for a free particle with a special preparation of initial conditions in the form of the Airy function [5].

\section{Liouville equation and FFPE}

A kinetic equation is an approximate way to describe an initial distribution, where, for a dynamical system, some details of the dynamics are neglected. Nevertheless, depending on the preserved information about the system, the type and specific structure of the kinetic equation depends on the choice of the reduced space of variables and the level of coarse-graining of trajectories. Therefore, this determines both the kinetic coefficients and transport exponents and, correspondingly, the rate of spreading of the initial distribution.

\subsection{Dynamics on phase space and Liouville equation}

First, we obtain the linear Liouville equation for $\left|\psi_{n}(t)\right|^{2} \equiv|\psi(n, t)|^{2}[15,16]$. Projecting Eq. (1.2) on the basis of the normal modes $\Psi_{k}(n)$

$$
\psi(n, t)=\sum_{\omega_{k}} C_{\omega_{k}}(t) \Psi_{\omega_{k}}(n) \equiv \sum_{k} C_{k}(t) \Psi_{k}(n),
$$

where $\Psi_{0}(n)=J_{n}(2 / E)$ (see Appendix A) and the translation invariance property $\Psi_{k+m}(n+m)=\Psi_{k}(m)$ $[21,42]$ is used, we obtain a system of equations for coefficients of the expansion $C_{k}$

$$
i \partial_{t} C_{k}=\omega_{k} C_{k}+\beta \sum_{k_{1}, k_{2}, k_{3}} A_{k_{2}, k_{3}}^{k, k_{1}} C_{k_{1}}^{*} C_{k_{2}} C_{k_{3}} .
$$


Here $A(\mathbf{k}) \equiv A_{k_{2}, k_{3}}^{k, k_{1}}$ is an overlapping integral of the four normal modes:

$$
A_{k_{2}, k_{3}}^{k, k_{1}}=\sum_{n} \Psi_{k}(n) \Psi_{k_{1}}(n) \Psi_{k_{2}}(n) \Psi_{k_{3}}(n)
$$

The initial conditions for the system of Eqs. (2.2) are such that $\psi^{(0)}(n)=\psi(n, 0)=\sum_{k} a_{k} \Psi_{k}(n)$ and $a_{k}=C_{k}(t=0)$. Equations $(2.2)$ correspond to a system of interacting nonlinear oscillators with the Hamiltonian

$$
H=\sum_{k} \omega_{k} C_{k}^{*} C_{k}+\beta \sum_{\mathbf{k}} A_{k_{2}, k_{3}}^{k_{1}, k_{4}} C_{k_{1}}^{*} C_{k_{4}}^{*} C_{k_{2}} C_{k_{3}} .
$$

Therefore, Eqs. (2.2) are produced by the Poisson brackets $\{H, \ldots\}$ PB by means of the Liouville operator

$$
\hat{L}=\frac{1}{i}\{H, \ldots\}_{\mathrm{PB}}=\frac{1}{i} \sum_{k}\left(\frac{\partial H}{\partial C_{k}^{*}} \cdot \frac{\partial}{\partial C_{k}}-\frac{\partial H}{\partial C_{k}} \cdot \frac{\partial}{\partial C_{k}^{*}}\right) .
$$

Since $\hat{L} H=0$ and $H\left(\left\{C, C^{*}\right\}\right)=H\left(\left\{a, a^{*}\right\}\right)$, we obtain that the Liouville operator is an operator function of the initial values

$$
\hat{L}=\frac{1}{i} \sum_{k}\left[\frac{\partial H}{\partial a_{k}^{*}} \cdot \frac{\partial}{\partial a_{k}}-\frac{\partial H}{\partial a_{k}} \cdot \frac{\partial}{\partial a_{k}^{*}}\right]
$$

and corresponds to an infinite system of linear equations $\partial_{t} \mathbf{C}=\hat{L} \mathbf{C}$, where $\mathbf{C}=\mathbf{C}\left(\left\{a_{k}, a_{k}^{*}\right\}\right)=$ $\left(\ldots, C_{k-1}, C_{k}, C_{k+1}, \ldots\right)$ is an infinite vector. Thus, the Liouville operator reads

$$
\hat{L}=-i \sum_{k} \omega_{k}\left(a_{k} \frac{\partial}{\partial a_{k}}-\text { c.c. }\right)-i \beta \sum_{\mathbf{k}} A_{k_{2}, k_{3}}^{k_{1}, k_{4}}\left[a_{k_{1}}^{*} a_{k_{2}} a_{k_{3}} \frac{\partial}{\partial a_{k_{4}}}-\text { c.c. }\right],
$$

where c.c. denotes the complex conjugation. The Liouville equation is valid for any functions of the initial conditions $\left\{a_{k}, a_{k}^{*}\right\}$. In particular, introducing the function $F_{k, k^{\prime}}(t)=C_{k}(t) \cdot C_{k^{\prime}}^{*}(t)$, one has the Liouville equation:

$$
\partial_{t} F_{k, k^{\prime}}(t)=\hat{L} F_{k, k^{\prime}}(t), \quad F_{k, k^{\prime}}(t=0)=F_{k, k^{\prime}}^{(0)}=a_{k} a_{k^{\prime}}^{*} .
$$

For convenience' sake, we consider the continuous limit $n a=x$, and, as will be seen, this does not influence the result of the analysis, since the localized normal modes are already used for inferring the system with the Hamiltonian (2.4). Therefore, the probability amplitude

$$
\mathcal{P}(x, t)=|\psi|^{2}=\sum_{k, k^{\prime}} F_{k, k^{\prime}}(t) \Psi_{k}(x) \Psi_{k^{\prime}}(x),
$$

as a function of the initial conditions, satisfies the Liouville equation as well:

$$
\partial_{t} \mathcal{P}=\hat{L} \mathcal{P} .
$$

Here the initial condition is

$$
\mathcal{P}(x, t=0)=\mathcal{P}_{0}(x)=\sum_{k, k^{\prime}} F_{k, k^{\prime}}^{(0)} \Psi_{k}(x) \Psi_{k^{\prime}}(x) .
$$

Finally, we obtain that the NLSE (1.2) is replaced by the Liouville equation (2.8), which is the linear equation with a formal solution in the exponential form

$$
\mathcal{P}(x, t)=e^{\hat{L} t} \mathcal{P}_{0}(x)=\sum_{k, k^{\prime}} \Psi_{k}(x) \Psi_{k^{\prime}}(x) \sum_{n=0}^{\infty}\left[\frac{t^{n}}{n !} \hat{L}^{n}\right] F_{k, k^{\prime}}^{(0)} .
$$


It is worth noting that the linear property of the Liouville equation (2.8) and its formal solution (2.10) are important for the probabilistic approach. The Liouville operator is, approximately, a sum of two operators $\hat{L}=\hat{L}_{0}+\hat{L}_{1}$, where $\hat{L}_{0}$ corresponds to the trapping determined by the overlapping integrals of the order of 1 , while $\hat{L}_{1}$ corresponds to jumps due to the exponentially small overlapping integrals. Thus one obtains $\hat{L}^{n} \approx \hat{L}_{0}^{n}+n \hat{L}_{0}^{n-1} \hat{L}_{1}$, where all commutations between $\hat{L}_{1}$ and $\hat{L}_{0}$ are neglected. Then using the identity $\sum_{m=0}^{n-1} 1=n$ and a definition of the beta function [34]

$$
\int_{0}^{1} y^{m}(1-y)^{n-1-m} d y=\frac{m !(n-1-m) !}{n !},
$$

we have from Eq. (2.10)

$$
\mathcal{P}(x, t)=e^{\hat{L}_{0} t} \mathcal{P}_{0}(x)+\int_{0}^{t} d t^{\prime} e^{\left(t-t^{\prime}\right) \hat{L}_{0}} \hat{L}_{1} e^{t^{\prime} \hat{L}_{0}} \mathcal{P}_{0}(x) .
$$

The probabilistic interpretation of the last expression is the following. The first term in Eq. (2.11) corresponds to a particle that at the initial time is at the position $x$ and there are no jumps until time $t$, and therefore $e^{\hat{L}_{0} t} \mathcal{P}_{0}(x) \rightarrow W(t) \mathcal{P}_{0}(x)$. Here $W(t)$ denotes the probability of no jump until time $t$. The last term in Eq. (2.11) corresponds to being at the position $x$ at time $t$ due to all possible jumps from $\left(x^{\prime}, t^{\prime}\right)$ with the transition probability $\mathcal{G}\left(x-x^{\prime} ; t-t^{\prime}\right)$, which is determined by operator $\hat{L}_{1}$. Note, also, that when $\beta=0$, the transition probability is also zero: $\mathcal{G}\left(x-x^{\prime} ; t-t^{\prime}\right)=0$. Therefore, Eq. (2.11) now reads

$$
\mathcal{P}(x, t)=W(t) \mathcal{P}_{0}(x)+\beta \int_{0}^{t} d t^{\prime} \int_{-\infty}^{\infty} d x^{\prime} \mathcal{G}\left(x-x^{\prime} ; t-t^{\prime}\right) \mathcal{P}\left(x^{\prime}, t^{\prime}\right) .
$$

This linear property can now be used for the continuous time random walks (CTRW) approach to obtain the Montroll-Weiss equation [27,31,32]. We also express here the dependence of the transition probability on $\beta$ in the explicit form to stress that, for $\beta \rightarrow 0$, the dynamics is localized. This dependence on $\beta$ will be reflected in a generalized transport coefficient. It should be admitted that CTRW processes are connected with a continuous time generalization of the Chapman-Kolmogorov equation [26, 44].

\subsection{Continuous time random walks}

In what follows, we consider the dynamics of the initial wave packet $\mathcal{P}_{0}(x)$ in the framework of the probabilistic, namely the CTRW, approach. Since the dynamics of the probability distribution function (PDF) $\mathcal{P}(x, t)$ in Eqs. (2.8) and (2.10) is governed by the same Liouville operator, the overlapping integrals $A(\mathbf{k})$ play a dominant role in the wave packet spreading. As follows from Eq. (2.10), the overlapping integrals determine the spread of the initially localized wave packet $\mathcal{P}_{0}(x)$ over all the normal modes as transitions from one mode to another. Since all states are localized, these transitions between states determine the transitions (or jumps) in the coordinate space as well.

We divide the transitions between the localized states into two main groups, according to the values of the overlapping integrals. The first one corresponds to the exponentially small overlapping integrals and the second one corresponds to the strong overlapping between four normal modes when the overlapping integrals are of the order of 1 . In the case of strong overlapping, the normal modes form clusters, where the wave functions have the same averaged coordinates for each cluster. Consequently, all transitions inside one cluster do not lead to any appreciable differences in the coordinate space, and we regard these transitions as trapping of the wave packet, or a particle, inside this cluster. On the contrary, transitions due to the exponentially small overlapping integrals between the normal modes belonging to different clusters lead to a change in the space coordinates that can be accounted for. We call these processes jumps. Contributions of trappings and jumps to the wave packet spreading described by Eqs. (2.8) and (2.10) are different, and correspond to different probabilistic interpretations.

In the sequel, we follow the CTRW approach [32] and paraphrase it from [4,27] in a form suitable for the present analysis. First, we consider a process of jumps. Let $P_{n}(x)$ be the PDF of being at $x$ after $n$ 
jumps. It is reasonable to assume that the transitions between different states are independent of each other; therefore, the jumps are independent and obey the Markov property

$$
P_{n+1}(x)=\int P_{n}\left(x^{\prime}\right) p\left(x-x^{\prime}\right) d x^{\prime},
$$

where $P_{0}(x)=\mathcal{P}_{0}(x)$ and $p(x)$ is the PDF of a jump determined by the overlapping integrals as $p(x)=$ $\xi \exp (-\xi|x|) / 2$, and $\xi=1 / R$ is an inverse localization length.

The trapping is associated with clusters with effective lengths $\Delta$. Due to the exponential localization, these values are distributed by the exponential law $P_{\mathrm{cl}}(\Delta)=\Delta_{0}^{-1} \exp \left(-\Delta / \Delta_{0}\right)$, where $\Delta_{0}$ is a characteristic length of a cluster. It is determined by overlapping integrals, as well; therefore, it is reasonable to take it as the maximum size of the cluster $\Delta_{0}=3 R$ (for four wave functions). Now the probability that a particle exits this cluster and jumps to another one is of the order of $\sim \exp (-\Delta / R)$. This value is also proportional to the inverse waiting time, $t \sim \exp (\Delta / R)$. The probability to find the waiting time in the interval $(t, t+d t)$ is equal to the probability to find the corresponding trapping length in the interval $(\Delta, \Delta+d \Delta)$, namely, $w(t) d t=P_{\mathrm{cl}}(\Delta) d \Delta$. Therefore, after simple calculations one obtains that the $\mathrm{PDF}$ of the waiting times is

$$
w(t)=P_{\mathrm{cl}}(\Delta) \frac{d \Delta}{d t} \sim \frac{1}{(t / \tau)^{1+\alpha}},
$$

where $\alpha=R / \Delta=1 / 3$ and $\tau$ is a scale parameter related to the trapping. It follows that the mean waiting time is infinite. Taking into account that the waiting time PDF is normalized we have

$$
w(t)=\frac{w_{0}}{1+(t / \tau)^{1+\alpha}},
$$

such that $\int_{0}^{\infty} w(t) d t=1$ and $\int_{0}^{\infty} t w(t) d t=\infty$. Here $w_{0}=\frac{2 \alpha \sin (\pi / 2 \alpha)}{\tau^{(1+\alpha) / 2 \alpha}}$ is a normalization constant.

Now let us consider the PDF $w(t)$ taking into account the dynamics of the jumps. Again, since transitions between different states are statistically independent, waiting times for different jumps are statistically independent as well. Therefore, indexing the waiting time PDF by the jump number, we define that $w_{n}(t)$ is the probability density that the $n$th jump occurs at time $t$ (see e.g., [4], p. 42). Due to the reasonable assumption that jumps are independent transitions, we also introduce the Markov property for $w_{n}(t)$, which reads

$$
w_{n+1}(t)=\int_{0}^{\infty} w_{n}\left(t^{\prime}\right) w\left(t-t^{\prime}\right) d t^{\prime},
$$

where $w_{1}(t) \equiv w(t)$. Now we introduce the PDF $P(x, t)=\sum_{n} P_{n}(x) w_{n}(t)$ of arriving at coordinate $x$ at time $t$. From Eqs. (2.13) and (2.16), we introduce the equation [27]

$$
\begin{aligned}
P(x, t) & =\int_{-\infty}^{\infty} p\left(x-x^{\prime}\right) \int_{0}^{\infty} w\left(t-t^{\prime}\right) P\left(X^{\prime}, t^{\prime}\right) d x^{\prime} d t^{\prime} \\
& +\mathcal{P}_{0}(x) \delta(t),
\end{aligned}
$$

which relates the PDF $P(x, t)$ of just having arrived at position $x$ at time $t$ to the PDF $P\left(x^{\prime}, t^{\prime}\right)$ of just arriving at $x^{\prime}$ at time $t^{\prime}$. The last term in Eq. (2.17) is the initial condition. Thus the PDF $\mathcal{P}(x, t)$ of being at position $x$ at time $t$ is given by arrival at $x^{\prime}$ at time $t^{\prime}$ and not having moved after this event, namely

$$
\mathcal{P}(x, t)=\int_{0}^{t} P\left(x, t^{\prime}\right) W\left(t-t^{\prime}\right) d t^{\prime},
$$

where $W(t)=1-\int_{0}^{t} w\left(t^{\prime}\right) d t^{\prime}$ denotes the probability of no jump during the time interval $(0, t)$ (cf. Sec. 2.1). Performing the Fourier transform $\bar{p}(k)=\hat{\mathcal{F}} p(x)$ and the Laplace transform $\tilde{w}(s)=\hat{\mathcal{L}} w(t)$, we obtain the Montroll-Weiss equation [32]

$$
\overline{\tilde{\mathcal{P}}}(k, s)=\hat{\mathcal{F}} \hat{\mathcal{L}} \mathcal{P}=\frac{1-\tilde{w}(s)}{s} \cdot \frac{\overline{\mathcal{P}}_{0}(k)}{1-\bar{p}(k) \tilde{w}(s)} .
$$


This equation can be simplified for the long time $s \ll 1$ and the large scale $k \ll 1$ asymtotics that corresponds to the diffusion limit $(k, s) \rightarrow(0,0)$. Taking into account the Fourier $\bar{p}(k)$ and the Laplace $\tilde{w}(s)$ images in Eq. (2.19),

$$
\begin{aligned}
& \bar{p}(k)=\frac{1}{1+\beta R^{2} k^{2}} \approx 1-\beta R^{2} k^{2}, \\
& \tilde{w}(s)=\frac{1}{1+(s \tau)^{\alpha}} \approx 1-(s \tau)^{\alpha},
\end{aligned}
$$

we obtain for the PDF in the Fourier-Laplace domain (see also [27])

$$
\overline{\tilde{\mathcal{P}}}(k, s)=\frac{\overline{\mathcal{P}}_{0}(k) / s}{1+D_{\alpha} s^{-\alpha} k^{2}},
$$

where $D_{\alpha}=\beta R^{2} / \tau^{\alpha}$ is a generalized diffusion coefficient. It should be stressed that the parameter $\beta$ in $\bar{p}(k)$ is introduced in such a way that for $\beta \rightarrow 0$ it follows $p(x) \rightarrow \delta(x)$, and there are no transitions for $\beta=0$.

Using the Laplace transform of the fractional integration (see Appendix B)

$$
\hat{\mathcal{L}}\left[\partial_{t}^{-\nu} f(t)\right]=\hat{\mathcal{L}} \frac{1}{\Gamma(\nu)} \int_{0}^{t} \frac{f(\tau) d \tau}{(t-\tau)^{1-\nu}}=s^{-\nu} f(s), \quad \nu>0,
$$

one obtains the fractional integral equation

$$
\mathcal{P}(x, t)-\mathcal{P}_{0}(x)=\partial_{t}^{-\alpha} D_{\alpha} \partial_{x}^{2} \mathcal{P}(x, t) .
$$

Differentiating this equation with respect to time, one obtains that the CTRW is described by the fractional Fokker-Planck equation (FFPE) ${ }^{1}$

$$
\partial_{t} \mathcal{P}(x, t)-D_{\alpha} \partial_{t}^{1-\alpha} \partial_{x}^{2} \mathcal{P}(x, t)=0 .
$$

Here $\partial_{t}^{\nu}$ is a designation of the Riemann-Liouville fractional derivative (see Appendix B)

$$
\partial_{t}^{\nu} f(t)=\frac{d}{d t} \partial_{t}^{\nu-1} f(t)=\frac{1}{\Gamma(1-\nu)} \frac{d}{d t} \int_{0}^{t} \frac{f(\tau) d \tau}{(t-\tau)^{\nu}}
$$

where $0<\nu<1$. From Eq. (2.23) one obtains for the second moment $\left\langle x^{2}(t)\right\rangle=\int_{-\infty}^{\infty} x^{2} \mathcal{P}(x, t) d x$ the differential equation

$$
\frac{d}{d t}\left\langle x^{2}(t)\right\rangle=\frac{2 D_{\alpha} t^{\alpha-1}}{\Gamma(\alpha)},
$$

where $\Gamma(z)$ is the gamma function, $x(t=0)=0$, and we use the following property of the fractional derivative $\partial_{t}^{\nu} 1=t^{-\nu} / \Gamma(1-\nu)$. Therefore, Eq. (2.23) describes subdiffusion

$$
\left\langle x^{2}(t)\right\rangle=\frac{2 D_{\alpha} t^{\alpha}}{\Gamma(1+\alpha)},
$$

with the transport exponent $\alpha=1 / 3$. In the numerical studies of the discrete NLSE $[11,21,36]$, the exponent $\alpha$ was found in the range $0.3 \div 0.4$ for $\beta \sim 1$.

\footnotetext{
${ }^{1}$ The solution of the FFPE is obtained in the form of the Fox function (see e.g., [27]) and its asymptotic behavior corresponds to the stretched Gaussian exponential function $\mathcal{P}(y) \sim \frac{1}{\sqrt{D_{\alpha} t^{\alpha}}} y^{-(1-\alpha) /(2-\alpha)} e^{-y^{2 /(2-\alpha)}}$, where $y \equiv \frac{|x|}{\sqrt{D_{\alpha} t^{\alpha}}} \gg$ 1. For $\alpha=1$, it corresponds to the normal Gaussian distribution.
} 


\section{Continuous limit of Eq. (1.2) and Painlevé II}

In what follows, we consider the continuous limit of Eq. (1.2). The situation with the continuous limit of Eq. (1.2) differs radically from the continuous limit introduced for convenience' sake in the preceding section. First, the linear part of the equation is described by the Airy function, and localization is absent. It is possible to show that, with a suitable variable change, the continuous NLSE is integrable and corresponds to the second Painlevé transcendent, expressed by expansion $\sum_{i=1}^{\infty} a_{i}\left(x-x_{0}\right)^{i}$ in the vicinity of a regular point $x_{0}[8]$.

\subsection{Stationary solutions of nonlinear Stark ladder equation}

Let us consider the continuous limit $x=n a$, and $\psi_{n}=\psi(x)$; correspondingly, we have

$$
\psi_{n \pm 1}=\psi(x) \pm a \psi^{\prime}(x)+\frac{a^{2}}{2} \psi^{\prime \prime}(x)+O\left(a^{2}\right),
$$

where $\psi^{\prime} \equiv \frac{d \psi}{d x}$ and $\psi^{\prime \prime} \equiv \frac{d^{2} \psi}{d x^{2}}$. The substitution of the stationary solution of $\psi_{n}=e^{-i \omega t} \phi(x)$ in Eq. (1.2) yields

$$
\omega \phi(x)=-\phi^{\prime \prime}+\operatorname{Ex} \phi(x)+\beta|\phi(x)|^{2} \phi(x) .
$$

The change in the parameters $\frac{\omega+2}{a^{2}} \rightarrow \omega$ and $\frac{E}{a^{2}} \rightarrow E$ is used, while for the nonlinear parameter one has $\frac{\beta}{a^{2}} \rightarrow \beta$. Here $\phi=\phi(x)$ is a complex function:

$$
\phi=R(x) \exp [i \theta(x)] .
$$

After inserting this expression for $\phi$ in Eq. (3.1), one obtains

$$
\omega R=-R^{\prime \prime}-2 i R^{\prime} \theta^{\prime}+R \theta^{\prime 2}-i R \theta^{\prime \prime}+x E R+\beta R^{3},
$$

which yields, after separation of the real and imaginary parts, the two equations

$$
\begin{gathered}
\omega R=-R^{\prime \prime}+R \theta^{2}+x E R+\beta R^{3}, \\
2 R^{\prime} \theta^{\prime}+R \theta^{\prime \prime}=0 .
\end{gathered}
$$

In the particular case $\theta(x)=0$, one obtains a kind of the Painlevé II equation [8]:

$$
R^{\prime \prime}+(\omega-x E) R-\beta R^{3}=0 .
$$

In the general case, we have from Eq. (3.5)

$$
\theta^{\prime}=A R^{-2}
$$

Substituting the solution in Eq. (3.4) yields the nonlinear equation

$$
\omega R=-R^{\prime \prime}+A^{2} R^{-3}+x E R+\beta R^{3} .
$$

To use the transformations suggested in Ref. [2], we denote $\rho(x)=\sqrt{\beta} R(x)$ and $\beta^{2} A^{2}=k^{2}$. Therefore we have from Eq. (3.3)

$$
\rho^{\prime \prime}=\frac{k^{2}}{\rho^{3}}+(E x-\omega) \rho+\rho^{3} .
$$

We then use the following variables change [2]

$$
E x-\omega=a z, \quad \rho(x)=b \sqrt{W(z)},
$$


where we have the relations

$$
\frac{2 a^{3}}{E^{2}}=-1, \quad \frac{2 k^{2} a^{2}}{b^{4} E^{2}}=-\frac{1}{2}, \quad a=-\left(\frac{E^{2}}{2}\right)^{\frac{1}{3}}, \quad b=\left[\frac{4 k^{2}}{-E^{2}} a^{2}\right]^{\frac{1}{4}} \quad 4 \alpha=\frac{2 b^{2} a^{2}}{E^{2}} .
$$

We obtain the following Eq. for $W(x)$ :

$$
\frac{d^{2} W}{d z^{2}}=\frac{1}{2 W}\left(\frac{d W}{d z}\right)^{2}+4 \alpha W^{2}-z W-\frac{1}{2 W} .
$$

This is Eq. XXXIV of Ref. [14], with the solution in the following differential form

$$
2 \alpha W=V^{\prime}+V^{2}+\frac{1}{2} z
$$

where the function $V$ is determined by the Painlevé II equation

$$
V^{\prime \prime}=2 V^{2}+z V-2 \alpha-\frac{1}{2}
$$

with the well defined solution in the Painlevé transcendent II form [8].

\subsection{Running Wave Solution}

Now let us look for a solution of Eq. (1.2) in the form of a running wave [41]

$$
\psi_{n}(t)=\exp \left[-i \omega t-i n a E t+2 i t-i \frac{t^{3} E^{2} a^{2}}{3}\right] W\left(n a+E a^{2} t^{2}\right) .
$$

Substitution of solution (3.14) in Eq. (1.2) yields the "stationary" equation in the continuous limit,

$$
(\omega-2) W=-a^{2} \frac{d^{2} W}{d y^{2}}+\beta|W|^{2} W
$$

where $y \equiv n a+a^{2} E t^{2}$. A general running wave solution is $W=\exp (i \theta(y)) R(y)$, which yields after substitution in Eq. (3.15)

$$
\begin{gathered}
\theta^{\prime}=A R^{-2}, \\
\bar{\omega} R=\frac{A^{2}}{R^{3}}-R^{\prime \prime}+\bar{\beta} R^{3},
\end{gathered}
$$

where $\bar{\omega}=\frac{\omega}{a^{2}}$ and $\bar{\beta}=\frac{\beta}{a^{2}}$. Again we use the change $\rho=\sqrt{\bar{\beta}} R$ and $-k^{2}=A^{2} \bar{\beta}^{2}$ to obtain

$$
\bar{\omega} \rho=-\frac{k^{2}}{\rho^{3}}-\rho^{\prime \prime}+\rho^{3} .
$$

Then we introduce new variable $y=a z$ and new function $\rho=b \sqrt{W}$ and define for the parameters $-\alpha=\frac{\bar{\omega}}{a^{2}}, b^{2}=2 a^{2}, \frac{k^{2}}{a^{2}}=\frac{1}{2}$. After substitution of these changes in Eq. (3.17), we obtain the following equation

$$
\frac{d^{2} W}{d z^{2}}=\frac{1}{2 W}\left(\frac{d W}{d z}\right)^{2}+4 W^{2}+\alpha W-\frac{1}{2 W},
$$

which is Eq. XXXIII in Ref. [14] with the first integral

$$
\left(\frac{d W}{d z}\right)^{2}=4 W^{3}+2 \alpha W^{2}+4 K W+1,
$$

where $K$ is an arbitrary constant. After substitution it yields a Pailevé transcendent I solution near a regular point $z_{0}[8]$

$$
W=\sum_{l=-2}^{\infty} a_{l}\left(z-z_{0}\right)^{l} .
$$




\section{Conclusion}

The nonlinear Schrödinger equation in the presence of an external field is considered for both discrete and continuous limits. This system exhibits different interesting regimes of spreading of an initial wave packet in both limits. A specific property of the discrete NLSE is localization of the linear counterpart (for $\beta=0$ ), and the dynamics of the initially localized wave packet was studied in this case. A subdiffusive spreading of the wave packed was observed and explained in the framework of the CTRW. The main mechanism of this subdiffusion is due to the transitions between those normal modes that are strongly overlapped. This overlapping leads to the clustering with an effective potential well and, correspondingly, to effective trapping of the wave packet inside each cluster by the effective cluster potential. Therefore, the dynamics of the wave packet corresponds to the CTRW, where the basic mechanism of subdiffusion is an entrapping of the wave packet with delay, or waiting, times distributed by the power law $w(t) \sim 1 / t^{1+\alpha}$, $0<\alpha<1$. We also have shown that for the nonlinear interaction $\beta|\psi|^{2 n}$, the transport exponent is $\alpha=1 / 3$ for $n=2$. Obviously, this result can be generalized for an arbitrary degree of the nonlinearity $n$ that yields

$$
\alpha=\frac{1}{2 n-1}
$$

The transport exponent $\alpha$ is independent of both the nonlinearity parameter $\beta$ and the bias $E$. It has pure topological nature, related to a coordination number $z=2 n-1$, and determines the maximal size of the trap potential, and the waiting time PDF in Eq. (2.14). Obviously, this trapping property is independent of the control parameters $\beta$ and $E$. Nevertheless, these parameters play an important role in the determination of a percolation threshold. For example, for the NLSE of Eq. (1.1), the percolation transition has been obtained for $\beta \approx 1.4$ [29] that corresponds to the localization-delocalization transition.

Two important properties of the constructed CTRW approach should be admitted. The first one is linearity of master equation (2.12) with $\mathcal{G}\left(x-x^{\prime} ; t-t^{\prime}\right)=w\left(t-t^{\prime}\right) p\left(x-x^{\prime}\right)$ that ensures asymptotic consideration in the framework of the Motroll-Weiss equation. Therefore, subdiffusion obtained in Eq. (2.24) is asymptotic. Another important property of the probabilistic consideration, developed in the framework of the CTRW, is a strong correlation effect determined by the waiting time pdf $w(t)$ in Eq. (2.15). This means that the dynamics of the underlying Hamiltonian system (2.4) is pseudochaotic [24], and subdiffusion takes place inside a complicated many dimensional (infinite dimensional) chaotic separatrix structure $[28,29]$. Therefore, this CTRW subdiffusion, connected with a weak breaking of ergodicity [13], also implies some restriction on the parameter $\beta$, since for large $\beta$, the nonlinear resonances are strongly overlapped, and strong chaos takes place, and correlation functions of dynamical variables decay exponentially fast $\langle C(t+\tau) C(t)\rangle \sim \exp (-h \tau)$ with the Kolmogorov-Sinai entropy $h$ [43]. The relevant kinetic description corresponds to the Markov process, and the developed CTRW approach, as a weak chaos admitted in $[10,29]$, is not valid.

The continuous counterpart of the biased NLSE (1.2) is integrable, and a chain of the variable changes reduces it to the Painlevé transcendents II and I. This transition to the continuous limit is accompanied by the transition from subdiffusion to the self-accelerated beam spreading. This solution corresponds to physical realizations of self-trapped and self-accelerating beam propagation in Kerr-like media $[18,19]$. As seen, this phenomenon also relates to the Airy function solution of the linear part of the equation.

Acknowledgements. I thank E. Yodim for language editing and N.N. Tarkhanov for useful discussions and comments. 


\section{A. Overlapping integrals}

As admitted in the main text, the overlapping integrals determine the transition probability in the master equation, and their property follows from the linear part of Eq. (1.2). When $\beta=0$, by the unsatz $\psi_{n}(t)=e^{-i \omega t} \Psi_{n}^{\omega}$ it reduces to the eigenvalue problem, which is the functional equation for the Bessel function of the first kind [17]:

$$
-\omega \Psi_{n}=-\Psi_{n+1}-\Psi_{n-1}+n a E \Psi_{n} .
$$

Here $\Psi_{n} \equiv \Psi_{n}^{\omega}$ and for an infinite lattice the eigenvalues corresponds to equidistant spectrum $\omega_{m}=E m$. One obtains from Eq. (A.1) $\Psi_{n}^{m}=J_{n-m}(2 / E a)$ that also obeys the Wannier translational invariance $\Psi_{n}^{m}=\Psi_{n+b}^{m+b}[42]$.

From the solution one can evaluate a localization length. First, we admit that when $E a \gg 1$, then $J_{n-m}(2 / E a) \sim(1 / E a)^{n}=e^{-\ln (E a) n}$ [17]. Therefore $\ln (E a)$ is the inverse localization length. One also uses the asymptotic expression for the Bessel function [35]

$$
J_{\nu}(\nu / \cosh \eta) \sim \frac{e^{-\nu(\eta-\tanh \eta)}}{\sqrt{2 \pi \nu \tanh \eta}} \quad(\nu \rightarrow \infty) .
$$

Here $|n-m|=\nu$ and $\frac{2}{E a}=\nu / \cosh \eta$. Now we consider $\eta>\tanh \eta$ and $n \rightarrow \infty$, and the weak electric field is applied 1/Ea 》1. As follows from Eq. (A.2), $\eta$ is, approximately, the inverse localization length. Indeed, we have that $(E a)^{-1} \sim n e^{-\eta}$, and for the intermediate asymptotics $1 / E a \gg 1$ and $1 / E a>n$, the inverse localization length is $\eta \sim|\ln (E a)|$. In the opposite case, when $n \gg 1 / E a$, one obtains a fast cut off

$$
\phi_{n}^{m} \sim n^{-n} \sim 1 / n ! .
$$

Therefore, the localization length is determined from the intermediate asymptotics for either cases $1 / E a \gg 1$ or $E a \gg 1$

$$
\eta \sim|\ln (E a)| .
$$

Taking into account this result, the overlapping integrals can be estimated as well

$$
A_{k_{2}, k_{3}}^{k_{1}, k_{4}}=\sum_{n} \Psi_{n}^{k_{1}} \Psi_{n}^{k_{2}} \Psi_{n}^{k_{3}} \Psi_{n}^{k_{4}} \approx \frac{1}{1-e^{-\eta}} e^{-\left(k_{1}+k_{2}+k_{3}+k_{4}\right) \eta}
$$

This expression determines the exponentially small transition probabilities of jumps between different clusters, while the overlapping integrals, which are of the order of 1 , determine the transition inside the same clusters.

\section{B. Fractional calculus briefly}

Fractional integration was developed as a generalization of integer order integration. Fractional integration of the order of $\alpha$ is defined by the operator (see e.g. $[27,34,38]$ )

$$
{ }_{a} I_{x}^{\alpha} f(x)=\frac{1}{\Gamma(\alpha)} \int_{a}^{x} f(y)(x-y)^{\alpha-1} d y,
$$

where $\alpha>0, x>a$ and $\Gamma(z)$ is the Gamma function. Fractional derivation is defined as the inverse operation to the fractional integral. Therefore, it is defined as the inverse operator to ${ }_{a} I_{x}^{\alpha}$, namely ${ }_{a} D_{x}^{\alpha} f(x)={ }_{a} I_{x}^{-\alpha} f(x)$ and ${ }_{a} I_{x}^{\alpha}={ }_{a} D_{x}^{-\alpha}$. Its explicit form is

$$
{ }_{a} D_{x}^{-\alpha} f(x)=\frac{1}{\Gamma(-\alpha)} \int_{a}^{x} f(y)(x-y)^{-1-\alpha} d y .
$$


For arbitrary $\alpha>0$, this integral diverges, and as a result of this a regularization procedure is introduced with two alternative definitions of ${ }_{a} D_{x}^{-\alpha}$. For an integer $n$ defined as $n-1<\alpha<n$, one obtains the Riemann-Liouville fractional derivative of the form

$$
{ }_{a} D_{R L}^{\alpha} f(x)=\frac{d^{n}}{d x^{n}}{ }_{a} I_{x}^{n-\alpha} f(x),
$$

and fractional derivative in the Caputo form (see also [25])

$$
{ }_{a} D_{C}^{\alpha} f(x)={ }_{a} I_{x}^{n-\alpha} f^{(n)}(x), \quad f^{(n)}(x) \equiv \frac{d^{n}}{d x^{n}} f(x) .
$$

There is no constraint on the lower limit $a$. For example, when $a=0$, one has

$$
{ }_{0} D_{R L}^{\alpha} x^{\beta}=\frac{x^{\beta-\alpha \Gamma(\beta+1}}{\Gamma(\beta+1-\alpha)}
$$

and

$$
{ }_{0} D_{C}^{\alpha} f(x)={ }_{0} D_{R L}^{\alpha} f(x)-\sum_{k=0}^{n-1} f^{(k)}\left(0^{+}\right) \frac{x^{k-\alpha}}{\Gamma(k-\alpha+1)},
$$

and ${ }_{a} D_{C}^{\alpha}[1]=0$, while ${ }_{0} D_{R L}^{\alpha}[1]=x^{-\alpha} / \Gamma(1-\alpha)$. When $a=-\infty$, the resulting Weyl derivative is

$$
{ }_{-\infty} \mathcal{W}^{\alpha} \equiv{ }_{-\infty} D_{W}^{\alpha}={ }_{-\infty} D_{R L}^{\alpha}={ }_{-\infty} D_{C}^{\alpha} .
$$

One also has ${ }_{-\infty} D_{W}^{\alpha} e^{x}=e^{x}$. This property is convenient for the Fourier transform

$$
\mathcal{F}\left[{ }_{-\infty} \mathcal{W}^{\alpha} f(x)\right]=(i k)^{\alpha} \bar{f}(k),
$$

where $\mathcal{F}[f(x)]=\bar{f}(k)$. The Laplace transform of the Caputo fractional derivative yields

$$
\mathcal{L}\left[{ }_{0} D_{C}^{\alpha} f(x)\right]=s^{\alpha} \tilde{f}(s)-\sum_{k=0}^{n-1} f^{(k)}\left(0^{+}\right) s^{\alpha-1-k},
$$

where $\mathcal{L}[f(x)]=\tilde{f}(s)$. We also use here a convolution rule for $0<\alpha<1$

$$
\mathcal{L}\left[I_{x}^{\alpha} f(x)\right]=s^{-\alpha} \tilde{f}(s) .
$$

Finally, this fractional derivation with the fixed low limit is also called the left fractional derivative. However, one can introduce the right fractional derivative, where the upper limit $a$ is fixed and $a>x$. For example, the right Weyl derivative is

$$
\mathcal{W}_{\infty}^{\alpha} f(z)=\frac{1}{\Gamma(-\alpha)} \int_{x}^{\infty} \frac{f(y) d y}{(y-x)^{1+\alpha}}
$$

\section{References}

[1] P.W. Anderson. Absence of diffusion in certain random lattices. Phys. Rev. 109 (1958), 1492-1505.

[2] Yu.Yu. Bagderina. Equivalence of ordinary differential equations. Differential Equations, 43 (2007), 595-604.

[3] R. Bekenstein, M. Segev, Self-accelerating optical beams in highly nonlocal nonlinear media. Optics Express, 19 (2011), No. 24, 23706-23715.

[4] D. ben-Avraam, S. Havlin. Diffusion and Reactions in Fractals and Disordered Systems. University Press, Cambridge, 2000.

[5] M.V. Berry, N.L. Balazs. Nonspreading wave packets. Am. J. Phys., 47 (1979) No. 3, 264-267.

[6] J. Billy, V. Josse, Z. Zuo, A. Bernard, B. Hambrecht, P. Lugan, D. Cl’ement, L. Sanchez-Palencia, P. Bouyer, A. Aspect. Direct observation of Anderson localization of matter waves in a controlled disorder. Nature, 453, (2008), 891-894. 
[7] B.V. Chirikov, V.V. Vecheslavov. Arnold diffusion in large systems. Sov. Phys. JETP, 85 (1997), 616 [Zh. Eksp. Teor. Fiz. 112 (1997), 1132].

[8] H.T. Davis. Introduction to Nonlinear Differential and Integral Equations. Dover Publications Inc., New York, 1962.

[9] D. Emin, C.F. Hart. Existence of Wannier-Stark localization. Phys. Rev. B, 36 (1987), 7353-7359.

[10] S. Flach. Spreading of waves in nonlinear disordered media. Chem. Phys., 375 (2010), 548-556.

[11] S. Flach, D.O. Krimer, Ch. Skokos. Universal spreading of wave packets in disordered nonlinear systems. Phys. Rev. Lett., 102 (2008), 024101.

[12] H. Fukuyama, R.A. Bari, H.C. Fogedby. Tightly bond electrons in a uniform electric field. Phys. Rev. B, 8 (1973), 5579-5586.

[13] Y. He, S. Burov, R. Metzler, E. Barkai. Random Time-Scale Invariant Diffusion and Transport Coefficients. Phys. Rev. Lett., 101 (2008), 058101.

[14] E.L. Ince. Ordinary Differential Equations. Longmans, Green and CO. LTD., London, 1927.

[15] A. Iomin. Subdiffusion in the nonlinear Schrödinger equation with disorder. Phys. Rev. E, 81 (2010), 017601.

[16] A. Iomin. Dynamics of wave packets for the nonlinear Schrödinger equation with a random potential. Phys. Rev. E, 80 (2009), 037601.

[17] E. Janke, F. Emde, F. Lösch. Tafeln Höherer Functionen. B.G. Taubner Verlagsgesellschaft, Stuttgart, 1960.

[18] I. Kramer, M. Segev, D.N. Christodoulides. Self-accelerating self-trapped optical beams. Phys. Rev. Lett., 106 (20110), 213903.

[19] I. Kramer, Y. Lamer, M. Segev, D.N. Christodoulides. Causality effects on accelerating light pulses. Optics Express, 19(23) (2011), 23132-23139.

[20] A.R. Kolovsky, E.A. Gómez, H.J. Korsh. Bose-Einstein condensates on tilted lattices: Coherent, chaotic, and subdiffusive dynamics. Phys. Rev. A, 81 (2010), 025603.

[21] D.O. Krimer, R. Khomeriki, S. Flach. Delocalization and spreading in a nonlinear Stark ladder. Phys. Rev. E, 80 (2009), 036201.

[22] Y. Lahini, A. Avidan, F. Pozzi, M. Sorel, R. Morandotti, D.N. Christodoulides, Y. Silberberg. Anderson localization and nonlinearity in one-dimensional disordered photonic lattices. Phys. Rev. Lett., 100 (2008), 013906.

[23] I.M. Lifshits, S.A. Gredeskul, and L.A. Pastur. Introduction to the Theory of Disordered Systems. Wiley-Interscience, New York, 1988.

[24] O. Lyubomudrov, M. Edelman, G.M. Zaslavsky. Pseudochaotic systems and their fractional kinetics. Intl. J. Modern Phys. B 17 (2003), 4149-4167.

[25] F. Mainardi. Fractional relaxation-oscillation and fractional diffusion-wave phenomena. Chaos Solitons Fractals, 7(9) (1996), 1461-1477.

[26] R. Metzler. Generalized Chapman-Kolmogorov equation: A unifying approach to the description of anomalous transport in external fields. Phys. Rev. E, 62 (2000), 6233-6245.

[27] R. Metzler, J. Klafter. The Random Walk's Guide to Anomalous Diffusion: a Fractional Dynamics Approach. Phys. Rep., 339 (2000), 1-77.

[28] A.V. Milovanov. Pseudochaos and low-frequency percolation scaling for turbulent diffusion in magnetized plasma. Phys. Rev. E, 79 (2009), 046403.

[29] A.V. Milovanov, A. Iomin. Localization-delocalization transition on a separatrix system of nonlinear Schrödinger equation with disorder. Europhys. Lett., 100 (2012), 10006.

[30] M.I. Molina. Transport of localized and extended excitations in a nonlinear Anderson model. Phys. Rev. B, 58 (1998), 12547.

[31] E.W. Montroll and M.F. Shlesinger. The wonderful wold of random walks. In Studies in Statistical Mechanics, v. 11, eds J. Lebowitz and E.W. Montroll. North-Holland, Amsterdam, 1984.

[32] E.W. Montroll, G.H. Weiss, Random walks on lattices. II J. Math. Phys., 6 (1965), 167; E.W. Montroll. Random walks on lattices III. Calculation of first passage times with application to exciton trapping on photosynthetic units. J. Math. Phys., 10 (1969), 753.

[33] M. Mulansky, K. Ahnert, A. Pikovsky, D.L. Shepelyansky. Strong and weak chaos in weakly nonintegrable many-body Hamiltonian systems. J. Stat. Phys., 145 (2011), 1256-1274.

[34] K.B. Oldham and J. Spanier. The Fractional Calculus. Academic Press, Orlando, 1974.

[35] F.W.J. Olver. Asymptotics and Special Function. Academic Press, New York, 1974.

[36] A.S. Pikovsky and D.L. Shepelyansky. Destruction of Anderson localization by a weak nonlinearity. Phys. Rev. Lett., 100 (2008), 094101.

[37] O.J. Pine, D.A. Weitz, P.M. Chaikin, E. Herbolzheimer. Diffusing wave spectroscopy. Phys Rev Lett., 60 (1988), 1134-1137.

[38] I. Podlubny. Fractional Differential Equations. Academic Press, San Diego, 1999.

[39] T. Schwartz, G. Bartal, S. Fishman, M. Segev. Transport and Anderson localization in disordered two-dimensional photonic lattices. Nature, 446 (2007), 52.

[40] D.L. Shepelyansky. Delocalization of quantum chaos by weak nonlinearity. Phys. Rev. Lett., 70 (1993), 1787.

[41] N.N. Tarkhanov, private communication.

[42] G.H. Wannier. Wave functions and effective Hamiltonian for Bloch electrons in an electric field. Phys. Rev., 117 (1960), 432-439.

[43] G.M. Zaslavsky. Statistical Irreversibility in Non- linear Systems. Nauka, Moscow, 1970.

[44] G.M. Zaslavsky. Fractional kinetic equation for Hamiltonian chaos. Physica D, 76, (1994), 110-122. 